\title{
Connections between Healthy Behaviour, Perception of Olive Oil Health Benefits, and Olive Oil Consumption Motives
}

\author{
Anita Silvana Ilak Peršurić *(D) and Ana Težak Damijanić (iD \\ Department Economics and Rural Development, The Institute of Agriculture and Tourism, 52440 Poreč, Croatia; \\ tezak@iptpo.hr \\ * Correspondence: anita@iptpo.hr; Tel.: +385-052-408-329
}

check for updates

Citation: Ilak Peršurić, A.S.; Težak Damijanić, A. Connections between Healthy Behaviour, Perception of Olive Oil Health Benefits, and Olive Oil Consumption Motives. Sustainability 2021, 13, 7630. https://doi.org/10.3390/su13147630

Academic Editor: Marian Rizov

Received: 29 May 2021

Accepted: 4 July 2021

Published: 8 July 2021

Publisher's Note: MDPI stays neutral with regard to jurisdictional claims in published maps and institutional affiliations.

Copyright: (c) 2021 by the authors. Licensee MDPI, Basel, Switzerland. This article is an open access article distributed under the terms and conditions of the Creative Commons Attribution (CC BY) license (https:// creativecommons.org/licenses/by/ $4.0 /)$.

\begin{abstract}
Olive oil has been consumed since ancient times and appreciated as a source of nutritious fats and as medical remedy, in addition to being praised in ceremonies. Today, when health is one of the top priorities of humankind, we examined what consumers believe and value about olive oil, which is generally considered a healthy product. Our survey examined the associations between the hedonic and health properties of olive oil, the motives important for olive oil consumption, and how these are influenced by healthy behaviour. There was clear evidence of the importance of culture, tradition, pleasure, olive oil health benefits, and healthy behaviour. There was a strong association between healthy behaviour and olive oil benefit perceptions and motives for olive oil consumption, with the olive oil benefits associated with the motives to consume olive oil.
\end{abstract}

Keywords: olive oil; health benefits; consumption; motives

\section{Introduction}

The image of olive oil is linked through centuries with positive descriptions, starting from the ancient Greeks, when Homer described olive oil as liquid gold and when old Greeks believed that the olive tree was created by the goddess Athena [1]. From these ancient times Mediterranean countries have a hereditary legacy of olive oil because it was an integral component of their daily diet and because they valued it for its nutritional properties. Additionally, it was used in ceremonies and festive occasions, consumed for its medicinal properties, and utilised for cosmetic purposes. Olive oil continues to be consumed as part of the human diet [2], mainly in countries around the Mediterranean Sea. Indeed, the "Mediterranean diet" is recognised worldwide as a healthy diet, hence, the increasing popularity of olive oil in other non-Mediterranean countries. Consequently, it may be assumed that there is a link between healthy behaviour (diet), health benefits, and consumption of olive oil.

\subsection{Olive Oil Consumption Motives}

The main reasons why consumers appreciate olive oil today, especially extra virgin olive oils, are associated with olive oil consumption motives, namely, its nutrition, health, and hedonic attributes.

The hedonic attributes of olive oil are usually related to the taste and smell during consumption, such as the appearance (colour), the aroma, the flavour, and the texture [3]. Since the visual appearance is the first attribute that can instantly affect consumers' acceptance or rejection of olive oil, the colour should meet consumers' preferences. Colour is a consequence of the pigments present (green, yellow), the proportion of which can vary according to the stage of maturity at harvest [4]. Olive oil can be consumed solely or added to food (such as salads, soups, fish, and vegetables) to improve the taste and texture of food in the mouth. Olive oil is distinct from other plant oils because of its specific olfactory qualities, such as fruitiness, astringency, and bitterness. These qualities can be detected in olive oils through the smell and taste of green or ripe olives, green leaves and 
grass, and aromatic herbs or those with a bitter or piquant taste [5]. Taste and smell are a consequence of the volatile aromas present in olive oils (which originate from polyphenols), which create bitterness, astringency, and pungency and which are also related to the health benefits of olive oil (the more polyphenols, the healthier the olive oil). For example, pungency is related to oleocanthal, a component with anti-inflammatory and antioxidant properties, while the throat-burning feeling relates to healthy components [6]. Thus, there is an association between the hedonic and health attributes of olive oil, as confirmed in several surveys [3].

Regarding the hedonic and health attributes of olive oil, previous studies also confirmed differences with respect to the consumption of olive oil between traditional and nontraditional olive-oil-consuming countries. In countries where olive oil is a non-traditional product, like Argentina [7], Brazil [8], Chile [9], and the UK [10], consumers' preferences relate to external attributes and hedonic and health dimensions that have proved to be highly important in consumer acceptance or rejection. The appreciation of extra virgin olive oil compared to other oils, especially in countries that do not traditionally consume olive oil, such as Argentina, show that certain attributes are highly validated, whereas others might lead to its rejection, for example, pungency and bitterness [6]. Furthermore, consumers in Argentina showed regional differences, with consumers in Buenos Aires not appreciating the olive oil attributes of pungency and bitterness, whereas respondents in the Mendoza region valued more olive oils with high sensory quality and recognised the differences between extra virgin and lampante oils.

Consumer surveys in traditional olive-oil-producing countries have determined the importance of the non-sensory aspects of olive oil, such as culture, tradition, and sustainability of production. Health properties were most important to organic olive oil consumers in Greece [11], primarily because of their culture, tradition, and knowledge, which provides consumers with awareness about the hedonic and health properties of olive oil. The exposure to olive oil consumption from childhood and the social conditioning to consume olive oil enhances not only the knowledge of children consumers but also creates positive future healthy behaviour and healthy consumption habits. From the perspective of sustainability, these future consumers provide a future market for olive oil producers in native countries (mostly in Mediterranean countries). Culture and tradition were also related to the consumption of organic oil, which is considered healthier [11] and which respects sustainability issues in the sense that the use of organic olive oil is environmentally friendly because of the minimal harmful substances used in production. Furthermore, culture and tradition as motives in consumer behaviour were linked to the issue of sustainable diets, which provide healthy locally grown food as well as economic sustainability for local farmers. Therefore, Greek consumers who were aware of harmful additives and pesticides in production favoured organic olive oil, small farm production, and environment protection. In this way, these consumers provide a market for sustainable farming, especially small-scale farming, and they ensure the long-term social and economic viability of these farms. Among the olive oil attributes, sensory (colour flavour, taste), utility (package, label, image), and health attributes were considered most important. By addressing the hedonic attributes, Greek consumers found colour and taste very important, which was supported by a survey conducted in Greece [12], which found that organic certification and origin were the most appreciated olive oil attributes.

In another traditional olive-oil-producing and -consuming country, Spain, a survey showed that consumers value extra virgin, organic, and low-priced oils, while another valued extra virgin olive oil, the origin (domestic), and the price and production system [13]. A case of different validation of hedonic and sensorial attributes of extra virgin olive oil among experts and average consumers in Italy [14] showed that experts highly valued the country of origin (Italy), protected origin, bottling in Italy, and sensorial features (fruity, pungent), whereas the market and consumers valued other attributes, especially sensorial features such as pungency and bitterness, which are not always appreciated by the average consumer but are highly validated by experts. 


\subsection{Olive Oil Health Benefits}

Olive oil health benefits are related to positive health claims or positive effects on health that relate to blood vessels' health, heart health, blood cholesterol levels, memory issues, metabolism, and cancer. The positive effects of olive oil and possible relations to lower risks of cardiovascular diseases were established in the findings about the Mediterranean diet [15-17]. According to a survey of health professionals (61 thousand women and 31 thousand men), the positive health effects of olive oil are associated with a lower risk of cardiovascular diseases [18]. Compared to non-consumers, olive oil consumption decreases the risk of cardiovascular disease by $14 \%$ and $18 \%$ for coronary heart diseases.

Regarding the quality attributes of olive oils, health claims ranked fifth, whereas sustainability was eleventh of the attributes evaluated [19]. The health benefits of olive oil relate to the heart and blood vessels, with positive effects on blood pressure, lowering cholesterol [20], anti-atherosclerosis and anti-obesity effects, psychological and memory effects (especially for Alzheimer disease), as well as the prevalence of rheumatoid arthritis. In the findings of [21], the use of animal fats was considered as the main reason for the development of inflammatory processes, hence, atherogenesis; therefore, the authors recommended switching from animal to plant fats as a way of diminishing levels of LDL and HDL cholesterol. Moreover, the authors suggested that olive oil may positively influence certain diseases such as atherosclerosis, coronary diseases, and breast cancer (in certain age and gender population segments).

Recently, the health benefits of olive oils have been attributed to the content of certain chemical components, such as polyphenols, quercetin, and oleocantal (which can be recognised in taste and smell). In the findings of [22], the effects of olive oil consumption on breast cancer incidence were negative (for postmenopausal women, the association was unlikely), whereas the link between total fat intake of saturated fat intake and cancer incidence was positive.

\subsection{Perception of Health Benefits and Knowledge of Olive Oil and Healthy Behaviour}

Among the hedonic attributes of food, foods may possess certain attributes that relate to health benefits. A study in the USA showed that $\frac{3}{4}$ of participants consumed olive oil exclusively because of its health benefits [23]. Several surveys had analysed how much the consumers understand what a health claim is and if certain foods have health benefits that may relate to diminishing risks for diseases or even curative effects of an existing disease. The perception of health benefits is a starting point for the realisation of health claim in consumer studies, that is, a statement about potentially positive effects of certain products on consumers' health [24]. Health claims describe positive effects and promise reduced risks for diseases, comprising three elements: the component that triggers the health function, the function as such, and the benefit derived from the physiological and psychological function [20]. Some consumers do not accurately understand the meaning of health claims [24], whereas others do [25]. A survey about heart health examined if consumers understood or accurately interpreted health claims and if their interpretation of health claims was mediated by their personal causal model of health [25]. The authors hypothesised that the strength of the benefit was influenced by the strength of the belief in and the familiarity with the claim and related to wider causal models of beliefs about overall health. They found that the participants had prior knowledge about heart health and their beliefs that fat and cholesterol affect heart health negatively. Moreover, participants related the presence of saturated fat in a diet to increased blood cholesterol levels, consequently exerting negative effects on heart health. Another survey in the UK [26] pointed out the influence of gender differences, whereby women tend to favour convenient, pleasurable, and healthy meals more than men. Their choices also relate to issues of body image and feeling guilty after food consumption, which, for women, are opposite to losing weight, obtaining a perceived ideal of a thinner body shape, and avoiding obesity. The consumption of a particular food, in this case, meat consumption, was linked not only to gender (men expressed that a healthy diet should always include meat) in the findings of [26] but it also 
expressed a traditional and masculine view of what is an appropriate healthy diet (for men, using animals for food is morally justified). The influence of tradition was evident in the views of women who considered a dinner consisting of meat, vegetables, and potatoes a base for a good diet, although women reported a lower frequency of meat consumption and more meal avoidance than men did. Additionally, $\frac{3}{4}$ of participants agreed or strongly agreed that information about healthy eating was widely available, although red meat was considered as a health risk (colon cancer, cholesterol, heart problems), and 5/6 felt confident about food that should be eaten in a healthy balanced diet.

There are regulations that have defined how health claims should be presented to avoid any misconceptions of health claims and to protect consumers. Since our survey was created and executed in Croatia and the sample contained EU citizens, the health claims were regulated by [20] EU legislation (EC No 1924/2006), whereas only claims that are substantiated by a review of the scientific evidence are allowed, and the claims should be understandable to the average consumer.

The discussions about healthy diets and food choices often pay attention about certain "bad" and "good" foods and beverages that should be included or excluded from the diet. Generally, fats, white sugars, and salt are marked as "bad," and, therefore, they are often excluded by persons who wish to follow healthy diet habits. The dietary fat content is minimised because fats are related to obesity, high blood cholesterol, and coronary diseases. Furthermore, saturated fats are considered "bad" for one's health; therefore, there is a general recommendation to switch from animal to plant fats, whereas many people consider switching saturated (animal fats) with unsaturated fats (plants fats, like the poly-unsaturated fats that are present in oils such as olive oil, rapeseed oil, and pumpkin oil). Since olive oil is considered healthy and recommended as part of a good diet for the prevention and cure of coronary diseases, it is regarded as a healthy fat. Olive oil is also promoted as healthy because of its antioxidant [27], nutritional [28], and non-saturated fat contents [21].

The perceived health and taste aspects of food were studied in Italy by [29], who focused on items such as general health interest and natural and light product interest, using sweet food as a reward. The authors discussed three consumers' clusters relating to health interest (low, medium, high) whereby the participants with the highest health interests expressed the lowest liking and frequency of consuming saturated and unsaturated fats, vitamins, and minerals. The motives for healthy food and diets were linked to balanced diets, avoiding increased cholesterol because of food intake, while avoiding additives and artificial flavourings in food. Their findings showed that older participants and women were more aware of healthy foods and healthy diets. Sweet food consumption was linked with the group of low-health-interest participants and those who desired instant gratification and pleasure from food (the stronger the craving for sweet food was, the weaker were the positive attitudes to health).

A multicultural study of consumers in west and southeast European countries on heart health evidence showed that consumers believe that the content of saturated fats in their diet is related to the increase in cholesterol and is negatively related to heart health and blood pressure [25]. Furthermore, consumers believed that high cholesterol was related to poor heart health, blood pressure, and increased weight. The duality of the findings in causal connections explained that consumers believed that both saturated fats and cholesterol negatively influence the heart and blood vessels and weight. Therefore, it was not explicitly clear if consumers can clearly distinguish the effects of saturated fats and cholesterol. Consumers believed that the effects of sodium in the relation to heart health were also negative, with sodium considered as a factor for increased body weight and blood pressure and high cholesterol [25].

Consumers' beliefs, motivations, and perceptions may explain what consumers believe the health benefits are and what they regard as healthy behaviour. Their beliefs, motivations, and perceptions are often associated with their knowledge, whereas there is a distinct difference in the explanations of knowledge and its influence on consumers' be- 
haviour; subjective knowledge explains what individuals perceive that they know, whereas objective knowledge explains what individuals know [30]. The relationship between subjective and objective knowledge is not straightforward and their correlation shows different effects because people do not accurately perceive how much or how little they know. These effects were explained in the case of subjective and objective knowledge and the consumption of organic foods by [31]. Their survey showed that a lack of knowledge about certain produce (in their case organic food) might lead to decreased consumption. The authors reported that consumers find organic products (vegetables) healthier (without pesticides) and better tasting, whereas, the price and availability were negatively related. The subjective knowledge was positively related to motivation and intention of consumption, affecting consumer behaviour, whereas the lack of knowledge was negatively related. In the case of organic food, objective knowledge was a consequence of beliefs and values, which created a perceived barrier and hindered consumption.

Based on the literature review, it was assumed that olive oil health benefits perception mediates the relationship between healthy diet and olive oil consumption motives (Figure 1). Seven hypotheses were proposed:

Hypothesis (H1). Health behaviour is positively linked to the perception of olive oil health benefits.

Hypothesis (H2). Health behaviour positively influence culture/tradition as a motive to consume olive oil.

Hypothesis (H3). Olive oil health benefits perception is positively linked to culture/tradition as a motive to consume olive oil.

Hypothesis (H4). Healthy behaviour positively influences pleasure as a motive to consume olive oil.

Hypothesis (H5). Olive oil health benefits perception is positively linked to pleasure as a motive to consume olive oil.

Hypothesis (H6). Olive oil health benefits mediate the relationship between health behaviour and culture/tradition as an olive oil consumption motive.

Hypothesis (H7). Olive oil health benefits mediates the relationship between health behaviour and pleasure as an olive oil consumption motive.

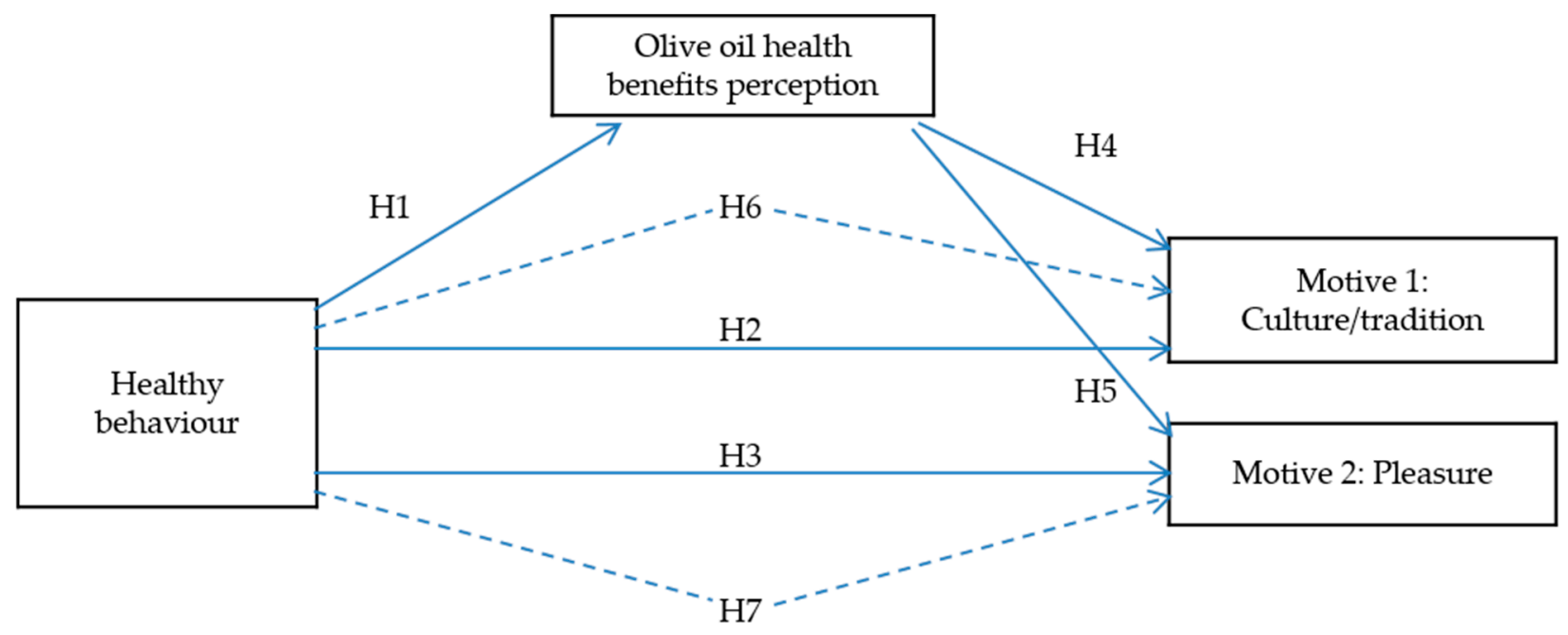

Figure 1. Theoretical framework. 


\section{Materials and Methods}

\subsection{Data Collection}

The target population included tourists who stayed in hotels located in Istria County, Croatia. The survey was conducted from June through September in 2019 in nine hotels situated in the towns of Novigrad, Poreč, Rovinj, and Umag. For the purpose of sampling, a list of accommodation facilities in Istria County [32] was obtained and hotels were categorised as either three- or four-star hotels (54 hotels in each category), with the hotels randomly selected based on hotel categorisation and capacity. The survey was conducted in ten hotels: six four-star hotels and four three-star hotels. Hotel guests were approached by the researcher and asked to participate in the survey. The researcher explained the purpose of the survey, stated that the survey was anonymous, and handed out a questionnaire in the appropriate language [33]. While the participants filled out the questionnaires, the researchers were available to address any queries or to provide additional information. Considering hotel capacity, every third (hotels with less than 350 rooms) or fourth (hotels with 350 or more rooms) hotel guest was approached.

Data was collected through a self-completed questionnaire that contained closed-type questions that had been piloted on a convenient sample of 35 Croatian consumers of olive oil to test clarity and understanding and to test statistical possibilities of the gathered data. After textual and technical adjustments, it was translated into foreign languages (Dutch, English, German, Italian, Slovenian, and Russian) by a certified translator. As a self-completed questionnaire was administered and because of the lack of tourist data, the sample size was based on the requirement for performing the data analysis [34]. Therefore, three levels were set: the minimal (300 responders), the optimal (500 respondents) and the maximal sample size (1000 respondents). Accordingly, sample sizes of 30 (minimal sample size), 50 (optimal sample size), and 100 (maximum sample size) responders per hotel were set.

\subsection{Data Analysis and Measurement}

Data was processed using statistical methods consisting of univariate, bivariate (chi square test and one-way ANOVA), multivariate (explorative factor analysis), and structural equation modelling. Descriptive statistics were used to provide a general description of the sample, whereas bivariate statistics were used to determine the differences between age, monthly spending for olive oil and olive oil consumption per month, and sample characteristics. Additionally, the relation between age and olive oil consumption motives, healthy behaviour, and olive oil benefits perceptions was examined in order to justify introductions of age as a control variable in the mediation model. Before establishing the structural model, exploratory and confirmatory factor analyses were conducted to test for the quality and adequacy of the measurement model by investigating reliability, convergent validity, and discriminant validity of the scale measuring olive oil consumption motives, the perceived benefits of olive oil, and healthy behaviour. The individual items were examined through checks for accuracy of data entry, missing data, and distribution. The cases with the missing values were replaced using the MCMC method for item imputation. The dataset was split randomly into two parts, so that the first part of the sample ( $20 \%$ of responders) was used to conduct the exploratory factor analysis, while the second part ( $80 \%$ of responders) was used to perform the confirmatory factor analysis. Exploratory factor analysis was performed using maximum likelihood factor analysis and promax rotation with an eigenvalue of 1.00 or more being used to identify potential factors. Internal reliability was determined by computing Cronbach's alpha. Exploratory factor analysis (EFA) was performed on 16 items measuring healthy behaviour and dietary habits adapted from $[29,35]$. The fourteen items measuring olive oil consumption motives were adapted from $[14,36]$, and six items measuring olive oil health benefits perception were adapted from $[21,27,28]$. The item knowledge about olive oil was measured similarly to the survey of [37]. Items with loading below 0.4 and cross-loadings were deleted, resulting in the retention of seven items measuring healthy behaviour and eleven items measuring olive 
oil consumption motives, while all items measuring olive oil health benefits perceptions were retained. Using eigenvalues greater than 1.0 as criteria, the two factors measuring olive oil consumption motives determined culture/tradition as an olive oil consumption motive (motive 1) and pleasure as an olive oil consumption motive (motive 2). Cronbach's alpha coefficients were 0.915 and 0.834 , respectively. The perceived benefits of olive oil and healthy diet were measured as a unidimensional construct, while healthy behaviour (diet) included seven items. Cronbach's alpha coefficients were 0.940 and 0.914 , respectively. All four factors accounted for $64.40 \%$ of the accumulated variance. The knowledge about olive oil was described by perceived knowledge or self-knowledge based on psychographic characteristics [9]. A Likert scale ranging from 1 to 5 was used to measure the judgements of self-estimated knowledge. For the mediation model, a bootstrapping analysis was run to test for mediation [38]. The maximum likelihood (ML) technique was applied in this study as the assumption of multivariate normality of the study data was confirmed.

\section{Results}

Most respondents spent up to $10 €$ per month (60\%) and consumed up to $5 \mathrm{~L}$ of olive oil, suggesting that they mostly bought olive oil of lower quality that might not be classified as a functional food (Table 1 ). Over $2 / 3$ of respondents were from countries that did not traditionally consume olive oil like Austria, Germany, the UK, and Russia. Most respondents were female $(60 \%)$, between 35 and 54 years of age $(51 \%)$, and employees $(53 \%)$. They had a higher education $(57 \%)$ with a personal monthly net income between $€ 1000$ and $€ 3000$ (57\%).

Country of origin $\left(\chi^{2}=129.452 ; \mathrm{df}=15 ;\right.$ sig. $=0.000$ and $\chi^{2}=187.077 ; \mathrm{df}=15 ;$ sig. $=0.000)$, profession $\left(\chi^{2}=78.118 ; \mathrm{df}=21 ;\right.$ sig. $=0.000$ and $\chi^{2}=39.811 ; \mathrm{df}=21$; sig. $=0.008)$, and age $(\mathrm{F}$ test $=9.932$ sig. $=0.000$ and $\mathrm{F}$ test $=4.471$ sig. $=0.004)$ were significantly related to monthly spending for olive oil and olive oil consumption per month, respectively. Responders from non-traditional olive oil consumption countries were less likely to spend more money on olive oil as well as to consume substantial amounts of olive oil per month compared to the responders from traditional olive oil consumption countries. Self-employed responders were more likely to spend more money on olive oil and to consume more quantities of olive oil compared to other professions. Older responders were more likely to consume higher quantities of olive oil and pay more for the oil compared to younger responders. Additionally, age was significantly correlated to olive oil health benefits, pleasure as an olive oil consumption motive, and healthy behaviour; therefore, it was used as a control variable in the mediation model.

\subsection{Confirmatory Factor Analysis}

The confirmatory factor analysis was performed to examine the reliability, the convergent validity, and the discriminant validity of the scale measuring olive oil consumption motives, perceived benefits of olive oil, and healthy diet (Table 2). The model fit indices for the measurement model, which were acceptable, and all indicators were significantly loaded onto their respective latent construct and were higher than 0.50. Each construct (Table 3) had an estimated composite reliability that exceeded the value of 0.60 and an extracted variance higher than the recommended threshold of 0.50 [31]. The results for the correlations showed that all measurement scales were interrelated. The correlations were all positive, and none of them were excessive. The final measurement model included a total of four factors: two factors measuring olive oil consumption motives (culture/tradition and pleasure), one factor measuring healthy behaviour, and one factor measuring olive oil health benefits. In general, culture/tradition was an important olive oil consumption motive (means varied from 4.0 to 4.1 ), whereas pleasure was a rather unimportant olive oil consumption motive (means varied from 2.6 to 2.9). Olive oil health perception benefits (means varied from 3.1 to 3.5 ) and healthy behaviour (means varied from 3.5 to 4.0 ) were somewhat important to responders. 
Table 1. Sample characteristics.

\begin{tabular}{|c|c|c|c|}
\hline Characteristic & Options & Frequency & Percentage \\
\hline \multirow[b]{2}{*}{ Gender } & Male & 262 & 39.6 \\
\hline & Female & 400 & 60.4 \\
\hline \multirow{6}{*}{ Age } & Up to 25 & 33 & 5.0 \\
\hline & 25 to 34 & 104 & 15.7 \\
\hline & 35 to 44 & 195 & 29.5 \\
\hline & 45 to 54 & 145 & 21.9 \\
\hline & 55 to 64 & 137 & 20.7 \\
\hline & 65 and more & 48 & 7.2 \\
\hline \multirow{7}{*}{ Country of origin } & Austria & 187 & 28.3 \\
\hline & Germany & 175 & 26.4 \\
\hline & UK & 109 & 16.5 \\
\hline & Russia & 31 & 4.7 \\
\hline & Croatia & 96 & 14.5 \\
\hline & Italy & 53 & 8.0 \\
\hline & Other & 11 & 1.6 \\
\hline \multirow{3}{*}{ Education level } & Primary school & 31 & 4.7 \\
\hline & High school & 251 & 37.9 \\
\hline & Higher education & 380 & 57.4 \\
\hline \multirow{5}{*}{ Profession } & Self-employed & 122 & 18.4 \\
\hline & Manager & 72 & 10.9 \\
\hline & Employee & 351 & 53.0 \\
\hline & Retired & 64 & 9.7 \\
\hline & Other & 53 & 8.0 \\
\hline \multirow{5}{*}{ Personal net monthly income } & Up to $€ 1000$ & 98 & 14.8 \\
\hline & $€ 1001-€ 2000$ & 206 & 31.1 \\
\hline & $€ 2001-€ 3000$ & 171 & 25.8 \\
\hline & $€ 3001-€ 4000$ & 97 & 14.7 \\
\hline & $€ 4001$ and more & 90 & 13.6 \\
\hline \multirow{4}{*}{ Monthly spending for olive oil } & Up to $€ 10$ & 399 & 60.2 \\
\hline & $€ 10-€ 20$ & 187 & 28.3 \\
\hline & $€ 21-€ 40$ & 50 & 7.6 \\
\hline & $€ 41$ and more & 26 & 3.9 \\
\hline \multirow{4}{*}{ Olive oil consumption per month } & $\mathrm{Up}$ to $1 \mathrm{~L}$ & 96 & 14.5 \\
\hline & $1 \mathrm{~L}$ to $2 \mathrm{~L}$ & 160 & 24.2 \\
\hline & $3 \mathrm{~L}$ to $5 \mathrm{~L}$ & 171 & 25.8 \\
\hline & More than $5 \mathrm{~L}$ & 235 & 35.5 \\
\hline
\end{tabular}

Table 2. Model of olive oil and healthy diet.

\begin{tabular}{ccccc}
\hline Factor & Item & Mean & SD & Standard Load \\
\hline Motive 1 & Olive oil consumption is a family tradition. & 4.1 & 1.11 & 0.939 \\
Culture & Olive oil consumption is part of one's cultural background. & 4.1 & 1.14 & 0.839 \\
/tradition & Extensive knowledge about olive oil. & 4.0 & 1.10 & 0.774 \\
\hline Motive 2 & Product is good for my health. & 2.9 & 1.45 & 0.786 \\
Pleasure & Olive oil is a natural product. & 2.7 & 1.47 & 0.783 \\
& Food smells and tastes better with olive oil. & 2.6 & 1.33 & 0.718 \\
\hline Olive oil & Olive oil provides health benefits. & 3.5 & 1.18 & 0.867 \\
benefits & Olive oil regulates blood cholesterol levels. & 3.5 & 1.17 & 0.774 \\
perception & Olive oil is good for my health. & 3.4 & 1.18 & 0.784 \\
& Olive oil is good for blood vessels' health. & 3.3 & 1.21 & 0.846 \\
Healthy & Olive oil is good for physical fitness and memory. & 3.1 & 1.27 & 0.769 \\
behaviour & Limitation of sugar intake. & 3.9 & 1.03 & 0.953 \\
& Limitation of fat intake. & 4.0 & 1.05 & 0.890 \\
& Limitation of salt intake. & 3.5 & 1.17 & 0.691 \\
& Increase in fibre intake. & 3.9 & 1.01 & 0.844 \\
\end{tabular}


Table 3. Scale reliability and discriminant validity.

\begin{tabular}{|c|c|c|c|c|c|c|}
\hline & CR & AVE & $\begin{array}{c}\text { Motive 1: } \\
\text { Culture/Tradition }\end{array}$ & $\begin{array}{l}\text { Healthy } \\
\text { Behaviour }\end{array}$ & $\begin{array}{l}\text { Olive Oil Benefits } \\
\text { Perception }\end{array}$ & $\begin{array}{l}\text { Motive 2: } \\
\text { Pleasure }\end{array}$ \\
\hline Motive 1: Culture/tradition & 0.807 & 0.582 & 0.763 & & & \\
\hline Healthy behaviour & 0.904 & 0.654 & $0.326^{* * *}$ & 0.809 & & \\
\hline Olive oil benefits perception & 0.914 & 0.683 & $0.622^{* * *}$ & $0.452^{* * *}$ & 0.826 & \\
\hline Motive 2: Pleasure & 0.889 & 0.728 & $0.558^{* * *}$ & $0.318^{* * *}$ & $0.541^{* * *}$ & 0.853 \\
\hline
\end{tabular}

Note: ${ }^{*}$ significant at $0.05,{ }^{* *}$ significant at $0.01^{* * *}$ significant at 0.001 ; bold figures in diagonal are the square root of the AVE.

\subsection{Findings of the SEM and Construct Relationships}

The relationships between the main constructs in the proposed model were then tested using an SEM (Figure 2), with the model fitting the data well (Table 4). Healthy behaviour (HB) was positively linked to olive oil benefits perception (OOBP) and motive 2 (M2) (pleasure as an olive oil consumption motive), while olive oil benefits perception was positively linked to motive 1 (M1) (tradition/culture as an olive oil consumption motive) and motive 2 . The study also tested the effect of the control variable, namely, responders age, with age statistically significant in relation to olive oil benefits perception $(\beta=0.111$, $p>0.01)$.

Table 4. Structural model results.

\begin{tabular}{ccccc}
\hline $\mathbf{H}$ & Relationship & $\boldsymbol{\beta}$ & $\mathbf{R}^{\mathbf{2}}$ & Decision \\
\hline H1 & Healthy behaviour $\rightarrow$ olive oil benefits perception & $0.447^{* * *}$ & 0.238 & Supported \\
H2 & Healthy behaviour $\rightarrow$ motive 1 & -0.033 & 0.529 & Not supported \\
H3 & Olive oil benefits perception $\rightarrow$ motive 1 & $0.732^{* * *}$ & & Supported \\
H4 & Healthy behaviour $\rightarrow$ motive 2 & $0.099^{* *}$ & \multirow{2}{*}{0.31} & Supported \\
H5 & Olive oil benefits perception $\rightarrow$ motive 2 & $0.503^{* * *}$ & & Supported \\
\hline
\end{tabular}

Note: $\chi^{2}=3.951 ; \mathrm{df}=1 ; p>0.05 ; \mathrm{CFI}=0.98 ; \mathrm{RMSEA}=0.05 ; \mathrm{RMSR}=0.03 ; \mathrm{TLI}=0.97 .{ }^{* *}$ significant at 0.01 $* * *$ significant at 0.001 .

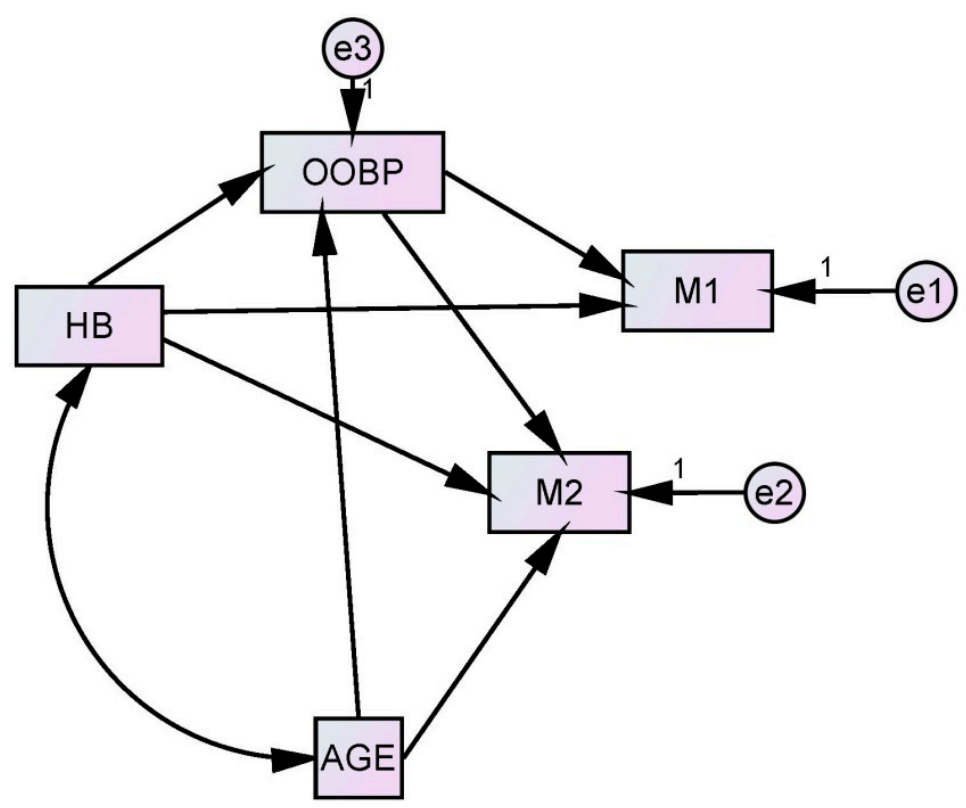

Figure 2. Mediation model.

The hypothesis that olive oil benefits perception mediates the relationship between healthy behaviour and olive oil consumption motives were then tested (Table 5). The 
bootstrapping analysis found that the indirect effect of healthy behaviour on motive 1 and healthy behaviour on motive 2 were both significant and positive.

Table 5. Indirect effects results.

\begin{tabular}{cccccc}
\hline H & Relationship & $\beta$ & \multicolumn{2}{c}{ Confidence Interval } & Decision \\
\cline { 4 - 5 } H6 & $\begin{array}{c}\text { Healthy behaviour } \rightarrow \text { olive oil } \\
\text { benefits Perception } \rightarrow \text { motive 1 } \\
\text { H7 }\end{array}$ & $0.316^{* * *}$ & 0.262 & 0.370 & Supported \\
$\begin{array}{c}\text { Healthy behaviour } \rightarrow \text { olive oil } \\
\text { benefits Perception } \rightarrow \text { motive 2 }\end{array}$ & $0.249^{* * *}$ & 0.205 & 0.309 & Supported \\
\hline Note: ${ }^{* * *}$ significant at 0.001.
\end{tabular}

\section{Discussion}

This study provided evidence that healthy behaviour was related to the association between hedonic and health benefits of olive oil. The use of olive oil provokes its hedonic perception and satisfaction of consuming a healthy product that may enhance a person's health. The image of olive oil as a natural product and its smell and taste were highly valued, as in [11]. The familiarity with the healthy compounds of olives was a prerequisite to olive oil consumption and healthy behaviour. Our survey provided evidence that consumers perceived olive oil as healthy for physical fitness and memory, particularly for blood vessel health. This is in accordance with the regulations [20], which state "plant sterols/stanols contribute to the maintenance of normal blood cholesterol in the development of human disease." Since consumers clearly recognised the health benefits of olive oil and perceived them as important, this fact may be used for future health claim legislation and regulations. Therefore, this study might be used as scientific evidence for developing further health claims for olive oil (such as in EC regulations [20]). The survey results could be used in the creation of packages and/or labels, which may boost the positive perception of olive oil as well as its perceived health benefits. Implicit visual images may attract more consumers to buy and consume olive oil and use it more often as part of healthy behaviour.

Since consumers were aware that fats should be limited in their diet, olive oil may be presented as a healthy alternative to other fats but used in moderation (the package/label might also indicate conscious use). This study examined the role of healthy behaviour on olive oil consumption motives and the mediation effects of olive oil health benefits perception on this relationship. The findings suggested that healthy behaviour supports olive oil consumption by directly influencing the motive to consume olive oil related to the product itself, i.e., the pleasure tourists get from the consumption of olive oil. However, contrary to the findings of [15], this study did not determine the influence of healthy behaviour to subjective knowledge about olive oil, which is evident from culture/tradition as an olive oil consumption motive. Additionally, olive oil health benefits mediate the relationship between healthy behaviour and both olive oil consumption motives, indirectly supporting the findings of how lifestyle influences consumption of functional foods [35] and supporting the finding that perceived product health benefits influence its consumption motivation [32]. The olive oil model showed similar results as the organic food one created by [36] in which food health attributes were found important and linked to health.

The study findings also provide insights for tourism and hospitality managers and to olive oil producers related to the importance of tourist's healthy behaviour on the consumption of olive oil, namely, on the olive oil product attributes and its health benefits. The tourism and hospitality facilities that promote niche tourism such as wellness and healthy lifestyles, sport, and gourmet tourism may incorporate olive oil as part of their nutrition and cuisine offering. For olive oil producers in Croatia and Istria, the tourism market offers sustainability of sales near the production site, especially for cold-pressed extra virgin olive oil and organic olive oil (similar to [39]). For tourists, the use of olive oil in different dishes during their vacation provides pleasure and the perception of doing well for one's health, which may stimulate its future use. Possible scenarios also include 
locally grown olive oils being bought as a souvenir and presented to family and friends as healthy gifts from the vacation destination.

Although the study helps to fill a gap in knowledge in the literature on this issue, there were some limitations. The results were based on a sample of tourists who stayed in selected hotels in Istria County; therefore, the results could not be generalised to Istria County's overall tourism market or to olive oil consumers in general. Future studies could include different populations (e.g., tourists in different accommodation facilities, olive oil family farm visitors), different parts of Croatia, and tourists staying in Croatia in the low and the pre/post seasons. Respondents were mostly tourists from countries that are not traditionally associated with olive oil production and consumption; therefore, future research may examine this model focusing mainly on tourists from traditional olive oil production countries such as Italy.

Author Contributions: A.S.I.P. was responsible for conceptualisation, validation, investigation; resources; writing — original draft preparation; and writing — review and editing; A.T.D. was responsible for methodology, software, formal analysis; writing — original draft preparation; writing — review and editing. Both authors have read and agreed to the published version of the manuscript.

Funding: This research received no external funding.

Data Availability Statement: Not applicable.

Acknowledgments: We would like to express our gratitude to olive oil consumers who patiently answered our questions during their stay in Istria in the period from June to September 2019. Special gratitude is given to the management of the hotels located in Istria County, Croatia that helped with the data gathering process.

Conflicts of Interest: The authors declare no conflict of interest.

\section{References}

1. Clodoveo, M.L.; Camposeo, S.; De Gennaro, B.; Pascuzzi, S.; Roselli, L. In the ancient world, virgin olive oil was called “liquid gold" by Homer and "the great healer" by Hippocrates. Why has this mythic image been forgotten? Food Res. Int. 2014, 62, 1062-1068. [CrossRef]

2. Corallo, A.; Latino, M.E.; Menegoli, M.; Spennato, A. A Survey to Discover Current Food Choice Behaviors. Sustainability 2019, 11, 5041. [CrossRef]

3. Tourila, H.; Recchia, A. Olive Oil Sensory Science, Chapter Sensory Perception and Other Factors Affecting Consumer Choice of Olive Oil; John Wiley \& Sons Ltd.: Hoboken, NJ, USA, 2014.

4. Moyano, M.J.; Heredia, F.J.; Meléndez-Martínez, A.J. The Color of Olive Oils: The Pigments and Their Likely Health Benefits and Visual and Instrumental Methods of Analysis. Compr. Rev. Food Sci. Food Saf. 2010, 9, 278-291. [CrossRef]

5. Peršurić, A.S.I. Segmenting Olive Oil Consumers Based on Consumption and Preferences toward Extrinsic, Intrinsic and Sensorial Attributes of Olive Oil. Sustainability 2020, 12, 6379. [CrossRef]

6. Beauchamp, G.K.; Keast, R.; Morel, D.; Lin, J.; Pika, J.; Han, Q.; Lee, C.-H.; Smith, A.B.; Breslin, P.A.S. Ibuprofen-like activity in extra-virgin olive oil. Nat. Cell Biol. 2005, 437, 45-46. [CrossRef] [PubMed]

7. Pardo, J.E.; Alvarez-Orti, M.; Araniti, V.; Banza, M.; Rubio, M.; Zied, D.C.; Silvestre, A. Argentinian consumers extra virgin olive oil preference. La Riv. Ital. Delle Sostanze Grasse 2018, 95, 105-110.

8. Antonialli, F.; Mesquita, D.L.; Valadares, G.C.; De Rezende, D.C.; De Oliveira, A.F. Olive oil consumption: A preliminary study on Brazilian consumers. Br. Food J. 2018, 120, 1412-1429. [CrossRef]

9. Romo-Muñoz, R.; Romo-Muñoz, F.; Dote-Pardo, J.S.; Troncoso-Sepúlveda, R. Incidence of psychographic variables on purchasing behavior in an emerging olive oil market. Br. Food J. 2018, 120, 2924-2936. [CrossRef]

10. Martínez, M.G.; Aragonés, Z.; Poole, N. A repositioning strategy for olive oil in the UK market. Agribusiness 2002, 18, 163-180. [CrossRef]

11. Sandalidou, E.; Baourakis, G.; Siskos, Y. Customers' perspectives on the quality of organic olive oil in Greece. Br. Food J. 2002, 104, 391-406. [CrossRef]

12. Krystallis, A.; Ness, M. Consumer preferences or quality foods from a South European Perspective: A conjoint analysis implementation on Greek Olive Oil. Int. Food Agribus. Manag. Rev. 2005, 8, 62-91.

13. Bernabéu, R.; Díaz, M. Preference for olive oil consumption in the Spanish local market. Span. J. Agric. Res. 2016, 14, e0108. [CrossRef]

14. Cavallo, C.; Caracciolo, F.; Cicia, G.; Del Giudice, T. Extra-virgin olive oil: Are consumers provided with the sensory quality they want? A hedonic price model with sensory attributes. J. Sci. Food Agric. 2017, 98, 1591-1598. [CrossRef] 
15. Bendinelli, B.; Masala, G.; Saieva, C.; Salvini, S.; Calonico, C.; Sacerdote, C.; Agnoli, C.; Grioni, S.; Frasca, G.; Mattiello, A.; et al. Fruit, vegetables, and olive oil and risk of coronary heart disease in Italian women: The EPICOR Study. Am. J. Clin. Nutr. 2010, 93, 275-283. [CrossRef]

16. Estruch, R.; Ros, E.; Salas-Salvadó, J.; Covas, M.A.; Corella, D.; Arós, F.; Gómez-Gracia, E.; Ruiz-Gutiérrez, V.; Fiol, M.; Lapetra, J.; et al. Primary Prevention of Cardiovascular Disease with a Mediterranean Diet Sup-plemented with Extra-Virgin Olive Oil or Nuts. N. Engl. J. Med. 2018, 379, 1388-1389.

17. Martinez-Gonzalez, M.A.; Dominguez, L.J.; Delgado-Rodríguez, M. Olive oil consumption and risk of CHD and/or stroke: A meta-analysis of case-control, cohort and intervention studies. Br. J. Nutr. 2014, 112, 248-259. [CrossRef]

18. Guasch-Ferré, M.; Liu, G.; Li, Y.; Sampson, L.; Manson, J.E.; Salas-Salvadó, J.; Martínez-González, M.A.; Stampfer, M.J.; Willett, W.C.; Sun, Q.; et al. Olive Oil Consumption and Cardiovascular Risk in U.S. Adults. J. Am. Coll. Cardiol. 2020, 75, 1729-1739. [CrossRef] [PubMed]

19. Perito, M.A.; Sacchetti, G.; Di Mattia, C.D.; Chiodo, E.; Pittia, P.; Saguy, I.; Cohen, E. Buy Local! Familiarity and Preferences for Extra Virgin Olive Oil of Italian Consumers. J. Food Prod. Mark. 2019, 25, 462-477. [CrossRef]

20. European Comission. Regulation of the European Parliaments and of the Council of 20 December 2006 on nutrition and health claims made on food. Off. J. Eur. Union 2006, 404, 9-25.

21. Harwood, J.L.; Yaqoob, P. Nutritional and health aspects of olive oil. Eur. J. Lipid Sci. Technol. 2002, 104, 685-697. [CrossRef]

22. The World Cancer Research Fund Report: Food, Nutrition, and the Prevention of Cancer; A Global Perspective; AICR: Washington, DC, USA, 2007.

23. Delgado, C.; Guinard, J.-X. Sensory Properties of Californian and Imported Extra Virgin Olive Oils. J. Food Sci. 2011, 76, S170-S176. [CrossRef]

24. Grunert, K.G.; Scholderer, J.; Rogeaux, M. Determinants of consumer understanding of health claims. Appetite 2011, 56, 269-277. [CrossRef] [PubMed]

25. Banks, A.P.; Egan, B.; Hodgkins, C.E.; Peacock, M.; Raats, M.M. The role of causal models and beliefs in interpreting health claims. Br. J. Heal. Psychol. 2018, 23, 933-948. [CrossRef] [PubMed]

26. Beardsworth, A.; Bryman, A.; Keil, T.; Goode, J.; Haslam, C.; Lancashire, E. Women, men and food: The significance of gender for nutritional attitudes and choices. Br. Food J. 2002, 104, 470-491. [CrossRef]

27. Owen, R.W.; Giacosa, A.; E Hull, W.; Haubner, R.; Würtele, G.; Spiegelhalder, B.; Bartsch, H. Olive-oil consumption and health: The possible role of antioxidants. Lancet Oncol. 2000, 1, 107-112. [CrossRef]

28. Stark, A.H.; Madar, Z. Olive oil as a functional food: Epidemiology and nutritional approaches. Nutr. Rev. 2002, 60, 170-176. [CrossRef]

29. Saba, A.; Sinesio, F.; Moneta, E.; Dinnella, C.; Laureati, M.; Torri, L.; Peparaio, M.; Civitelli, E.S.; Endrizzi, I.; Gasperi, F.; et al. Measuring consumers attitudes towards health and taste and their association with food-related life-styles and preferences. Food Qual. Prefer. 2019, 73, 25-37. [CrossRef]

30. Brucks, M. The effects of product class knowledge on information search behaviour. J. Consum. Res. 1985, 12, 1-16. [CrossRef]

31. Aertsens, J.; Mondelaers, K.; Verbeke, W.; Buysse, J.; Van Huylenbroeck, G. The influence of subjective and objective knowledge on attitude, motivations and consumption of organic food. Br. Food J. 2011, 113, 1353-1378. [CrossRef]

32. Tourist Board of Istria. 2018. Available online: http:/ / www.istra.hr/hr/smjestaj (accessed on 25 March 2019).

33. Veal, A.J. Research Methods for Leisure and Tourism: A Practical Guide; Pearson Education Limited: Essex, UK, $2006 ;$ p. 446.

34. Hair, J.F.; Black, W.C.; Babin, B.J.; Anderson, R.E. Multivariate Data Analysis, 7th ed.; Pearson Education Inc.: London, UK, 2010; p. 761.

35. Cicerale, S.; Liem, G.; Keast, R.S. Consumer Perception, Attitudes, Liking and Preferences for Olive Oil. Prod. Olive Tree 2016, 157-172. [CrossRef]

36. Samoggia, A.; Riedel, B. Consumers' Perceptions of Coffee Health Benefits and Motives for Coffee Consumption and Purchasing. Nutrients 2019, 11, 653. [CrossRef] [PubMed]

37. Salazar-Ordóñez, M.; Rodríguez-Entrena, M.; Cabrera, E.R.; Henseler, J. Understanding product differentiation failures: The role of product knowledge and brand credence in olive oil markets. Food Qual. Prefer. 2018, 68, 146-155. [CrossRef]

38. Hayes, A. Introduction to Mediation, Moderation, and Conditional Process Analysis: A Regression-Based Approach; Guilford Publications: New York, NY, USA, 2013; pp. 1-692.

39. Rizzo, G.; Borrello, M.; Guccione, G.D.; Schifani, G.; Cembalo, L. Organic Food Consumption: The Relevance of the Health Attribute. Sustainability 2020, 12, 595. [CrossRef] 